\title{
A case of retropharyngeal emphysema
}

\author{
Tsuyoshi Suda ${ }^{1}$, Tomoaki Yoneda ${ }^{2}$, and Yukari Ichikawa ${ }^{1}$ \\ ${ }^{1}$ Kanazawa Municipal Hospital \\ ${ }^{2}$ Kanzawa Municipal Hospital
}

May 6, 2020

\begin{abstract}
Retropharyngeal emphysema is a rare condition with various causes including traumatic and iatrogenic injuries, or it could occur spontaneously. Symptoms of mediastinitis and/or airway obstruction should be evaluated as indicators for surgical incision. Complications of mediastinitis are associated with poor prognosis; therefore, worsening of symptoms should be carefully monitored.
\end{abstract}

\section{Clinical Image:}

A 73-year-old man with moderate pulmonary emphysematous changes developed left-sided pneumothorax and dyspnea. Marked subcutaneous emphysema was observed on the neck and left anterior chest, along with facial disfiguration (Figure 1). Computed tomography revealed retropharyngeal, mediastinal, and subcutaneous emphysema. The emphysema involved the left anterior chest, bilateral neck regions, the surrounding temporal muscles, and the posterior region. Furthermore, pneumomediastinum, extending to the laryngopharynx, and air in the retropharyngeal space were radiographically observed (Figure 2). Based on these results, the patient was diagnosed with retropharyngeal emphysema. Fortunately, he did not show symptoms of airway obstruction. A chest drain was placed for treatment, and drainage was continued. Following drainage, the pneumothorax improved, and the retropharyngeal, mediastinal, and subcutaneous emphysema resolved completely.

Retropharyngeal emphysema can result from severe maxillofacial injuries or traumatic injury to the pharynx or esophagus, or it can occur spontaneously. Moreover, it can also be caused by iatrogenic factors such as dental procedures or tonsillectomy. ${ }^{1}$

Surgical incision and drainage are often indicated for mediastinitis or symptoms of airway obstruction. ${ }^{1}$ Complications of mediastinitis are associated with poor prognosis. ${ }^{2}$ The appearance of worsening dyspnea, chest pain, abdominal pain, or signs and symptoms related to shock should be carefully monitored. ${ }^{2}$

\section{Figure Legends}

Figure 1. Marked subcutaneous emphysema on the neck and left anterior chest, with facial disfiguration.

Figure 2. Radiograph of the neck showing pneumomediastinum, extending to the laryngopharynx, and air in the retropharyngeal space.

\section{Authorship}


TS cared for the patient, conducted the literature search, edited the manuscript, and prepared the figure. TY contributed to the editing of the manuscript and preparation of the figure. IY cared for the patient, contributed to the editing of the manuscript and provided expert opinion on pulmonology.

\section{References}

[1] Daniel A., and Onakoya P. A. 2013. Retropharyngeal emphysema: An unusual aetiology-A case report and literature review. Case Rep Clin Med 2:194-197.

[2] Cho, D.Y., Aaron, G.P., Shepard, K.G. 2016. Spontaneous retropharyngeal and mediastinal emphysema. Clin Exp Otorhinolaryngol 9:178-181.

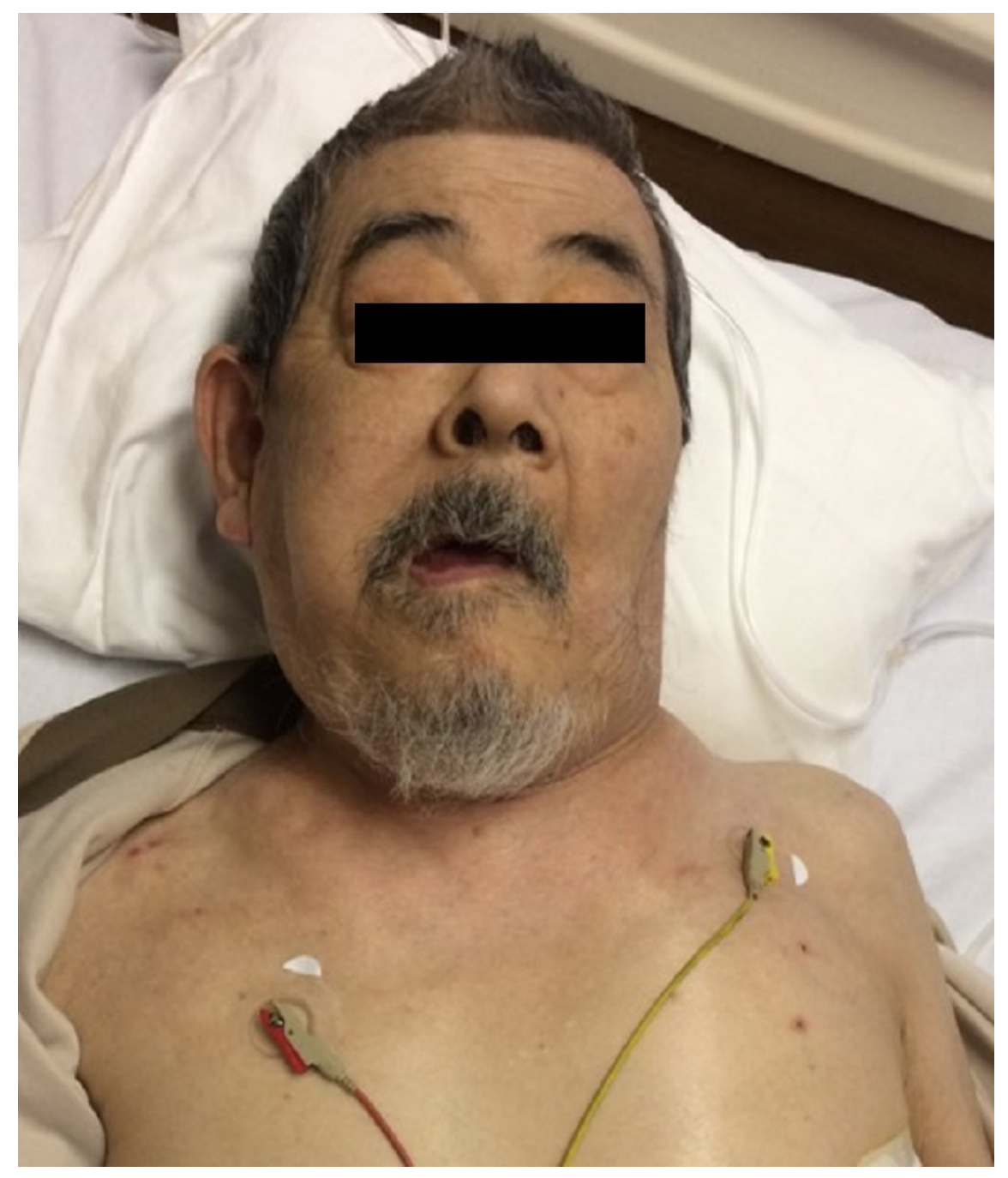




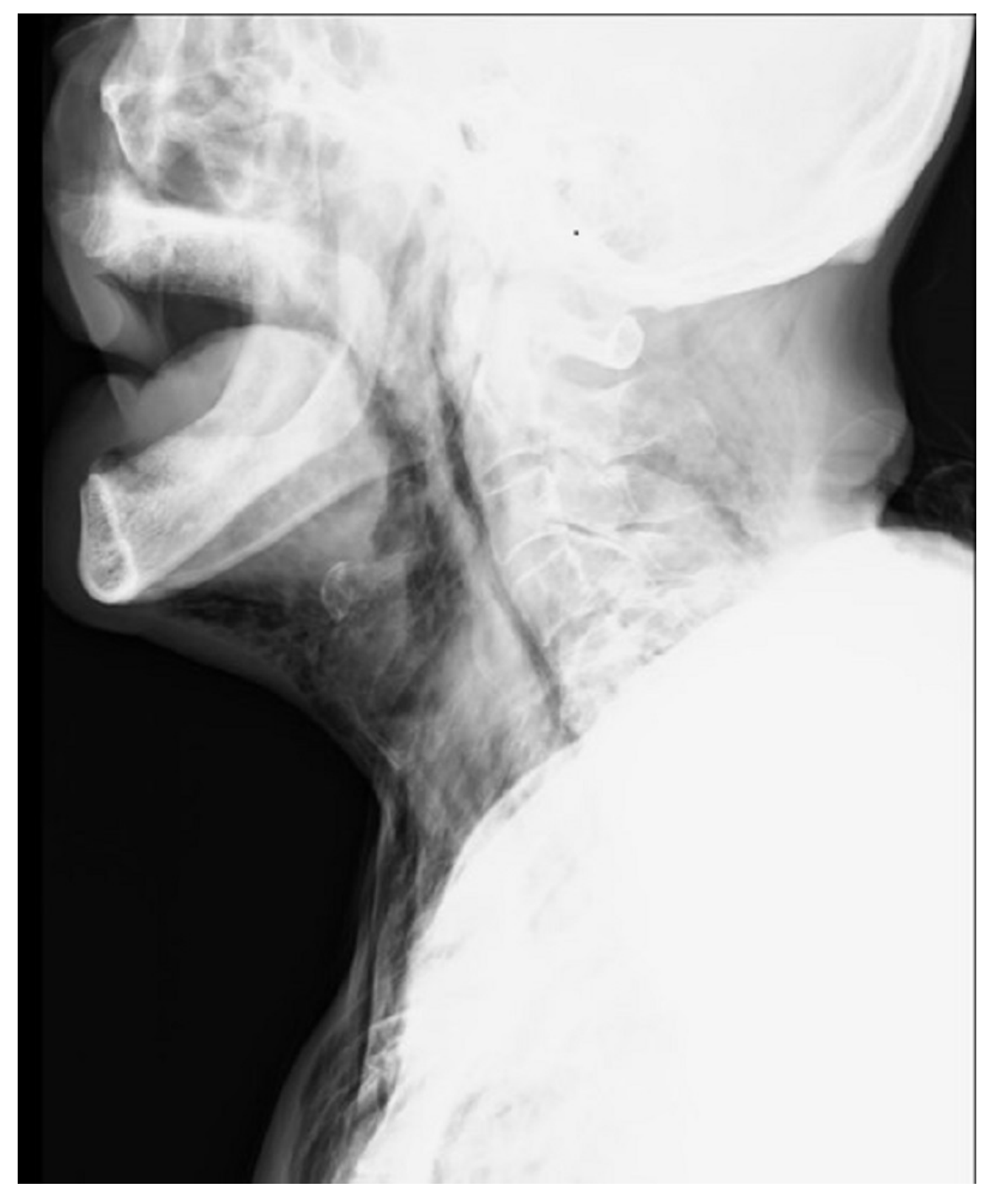

